\title{
Noninvasive imaging vulnerable plaques of atherosclerosis: Antiatherogenic properties of dietary flaxseed
}

\author{
Vanita S. Bhat ${ }^{1}$ and Basavaraj Madhusudhan ${ }^{2 *}$ \\ ${ }^{1}$ Department of Food Technology, Davangere University, India \\ ${ }^{2}$ Department of Biochemistry and Food Technology, Davangere University, India
}

\begin{abstract}
Atherosclerosis is an inflammatory disease complicated by progressively increasing atherosclerotic plaques in the arterial walls. The life- threatening syndrome adversely affects the arterial blood vessels in response to chronic inflammatory signaling of white blood cells in the walls of arteries to form multiple atheromatous plaques. To date, several methods of identification of high-risk atherosclerotic invasive and noninvasive plaque imaging modalities and biomarkers in high-risk patients is warranted. Intracoronary imaging strategies for confirmation of plaque existence and characteristics are detectable in humans. More recently, computed tomography (CT) or electron beam tomography (EBT) and gray-scale intravascular ultrasound (IVUS) imaging are used to visualize the external elastic lamina of the vessel wall to determine the vessel size, plaque components and morphology in comparison to angiography. Further, the beneficial effects of radiofrequency (RF) analysis of the ultrasonic signal (RF-IVUS) would be helpful to distinguish the nature of composition among fibrotic plaque and fibro-calcific plaque of thick-cap fibro-atheroma (TCFA) in vulnerable plaques of atherosclerosis. Akin to pharmaceuticals, functional foods can also be used to prevent and treat cardiovascular diseases. Recently, the beneficial effects dietary flaxseed on the regression of atherosclerotic plaques or the resolution of cholesterol-induced vascular contractile dysfunction has been well documented.
\end{abstract}

\begin{abstract}
Abbreviations: CT: Computed Tomography; EBT: Electron Beam Tomography; IVUS: Intravascular Ultrasound; RF: Radiofrequency; TCFA: Thick-Cap Fibro-Atheroma; PAD: Peripheral Arterial Disease; IMT: Intimal Media Thickness; PET: Positron Emission Tomography; SPECT: Single Photon Emission Computed Tomography; CVD: Cardiovascular Disease; ALA: $\alpha$-Linolenic Acid; PUFA: Polyunsaturated Fatty Acid
\end{abstract}

\section{Vulnerable plaque is considered to be the susceptibility of a plaque to rupture}

Atherosclerosis is the cause of more than $50 \%$ mortality in industrial countries. Worldwide life-threatening atherosclerosis or hardening of the arteries has become a major cause of thoracic aorta atherosclerosis or abdominal aortic aneurysm leading to coronary artery disease, stroke, and peripheral arterial disease (PAD). This chronic disease appears to be asymptomatic and remains for decades unnoticed. Among vascular risk factors, smoking and hypercholesterolemia are associated with severe aortic arch plaques. The major clinical manifestations of this vascular disease at the global level include ischemic heart disease, ischemic stroke, and peripheral arterial disease. The leading promoters in hardening of the arteries none other than low-density lipoproteins linked with inadequate removal of fats and cholesterol from the macrophages by functional high-density lipoproteins. The plaques are known to be in two different forms viz., stable and unstable (vulnerable) found in any given blood vessel [1]. Many factors are responsible to cause of atherosclerosis, normally associated with high blood pressure and cholesterol from low-density lipoproteins begins to damage the endothelium allowing forming plaques in the wall of the arteries that are prone to rupture. In most cases, severe and debilitating events suddenly occur due to rupture of plaques leading to artery lumen occlusion within seconds to minutes, and sometimes sudden death [2].

\section{Diagnosis of atherosclerotic vulnerable plaques}

Generally, plaque is divided into three distinct components including the atheroma of yellowish, soft and flaky calcified material surrounded by the macrophages embedded cholesterol crystals, presenting the picture of an early phase of arteriosclerosis by stiffening the arteries [3]. Routinely, the use of traditional methods of detection of atherosclerotic disease like angiography and stress-testing are being followed in addition to anatomical detection and physiologic measurement for the past few decades. Most of the times, it is difficult to locate the calcified deposits and areas of severe narrowing (stenosis) on coronary artery walls by angiography to obtain anatomical information. In order to support, the relatively advanced computed tomography (CT) or electron beam tomography (EBT), carotid IMT (intimal media thickness) measurement by ultrasound, and intravascular ultrasound (IVUS) have made possible to visualize and demonstrate unequivocal evidence of the disease due to rings of increased radiographic density that have formed halos around the outer edges of the atheromatous. Simultaneously, physiologic measurement such as lipoprotein subclass analysis, HbA1c (hemoglobin A1c), hs-CRP (high-sensitivity C-reactive protein) and homocysteine are considered to correlate the severity of the staging and tracking of disease progression. Usually, anatomic methods are more expensive and few of them are invasive in nature include IVUS in comparison with much safer physiologic

Correspondence to: Basavaraj Madhusudhan, Professor and Chairman, Department of Biochemistry and Food Technology, Davangere University, India, Tel: +91-9880548239; E-mail: prof.madhusudhan@gmail.com

Key words: imaging, plaques, atherosclerosis, flaxseed

Received: July 17, 2017; Accepted: August 17, 2017; Published: August 21, 2017 
methods. Unfortunately, physiologic methods are unable to provide the magnitude of the disease and its progression. In recent years, the nuclear imaging techniques such as positron emission tomography (PET) and single photon emission computed tomography (SPECT) appears to most promising and have provided ways of estimating the severity of atherosclerotic plaques [4]. In PET, radioactive isotopes with short half-lives are used include carbon-11 ( $20 \mathrm{~min})$, nitrogen-13 ( 10 min), oxygen-15 ( 2 min) or rubidium $-82(\sim 1.27 \mathrm{~min})$.

\section{Medication and the role of dietary flaxseed in regres- sion of atherosclerotic plaques}

Inflammatory areas in vascular region are the key targets for therapy in atherosclerosis. The medication of statins is recommended to block the action of the liver enzyme responsible for cholesterol synthesis and slow the progression of atherosclerosis in addition to unwanted side-effects. Though statins work as wonder molecules with potent anti-inflammatory properties, oral therapy poses low systemic bioavailability. In recent days, researchers are working on injectable reconstituted high-density lipoprotein nanoparticle carrier vehicle to deliver statins to the targeted atherosclerotic plaques [5]. Most recently, nanoformulations of multifunctional dextran sulfatecoated reconstituted high-density lipoproteins are promising not only target macrophages but to prevent macrophage-derived foam cell formation and inflammation by intracellular lipid deposition, cholesterol esters content, damping oxidized low-density lipoproteins uptake, promoting cholesterol efflux and secretion of TNF- $\alpha$, IL- 6 and IL-10 [6]. Unfortunately, tachyphylaxis, long-term toxicity and cost amongst other issues may present problems for the use of conventional medications in the long-term. Drugs from natural sources are gaining attention as a good alternative to statins. Our research group is engaged in developing a series of nutraceutical curcumin-loaded formulations using nanotechnological approach that are specifically designed to act at the site of vessel wall to disrupt the plaques and modulate the atherosclerotic lesion [7,8]. Functional foods, such as flaxseed, have increased in popularity and are now considered to be a viable therapeutic strategy for CVD [9]. Flaxseed contains one of the highest contents of $\alpha$-linolenic acid (ALA), a $\omega-3$ polyunsaturated fatty acid (PUFA) linked with CVD prevention [10].

\section{Future perspective}

The results of various drug therapies in comparison with $\omega-3$ PUFAs and lignan components have fewer and milder side effects and are generally considered safe [11]. There are ample of research evidences that have utilized nutritional interventions to induce plaque regression, these studies have all prematurely initiated their interventions before there is clear evidence of cessation of plaque growth [12]. It is evident that the anti-atherosclerotic effects of drugs based on flaxseed $\alpha$-linolenic acid and lignans are not inferior to the effects of such drugs as statins and calcium antagonists.

\section{Conflict of interest}

There are no conflicts of interest in the content of this paper.

\section{References}

1. Sharma K, Kumar K, Mishra N (2016) Nanoparticulate carrier system: a novel treatment approach for hyperlipidemia. Drug Deliv 23: 694-709. [Crossref]

2. Libby P, Ridker PM, Hansson GK (2011) Progress and challenges in translating the biology of atherosclerosis. Nature 473: 317-325. [Crossref]

3. Cecelja M, Chowienczyk P (2016) Molecular Mechanisms of Arterial Stiffening. Pulse (Basel) 4: 43-48. [Crossref]

4. Taqueti VR, Dorbala S (2016) The role of positron emission tomography in the evaluation of myocardial ischemia in women. J Nucl Cardiol 23: 1008-1015. [Crossref]

5. Duivenvoorden R, Tang J, Cormode DP, Mieszawska AJ, Izquierdo-Garcia D, et al. (2014) A statin-loaded reconstituted high-density lipoprotein nanoparticle inhibits atherosclerotic plaque inflammation. Nat Commun 5: 3065. [Crossref]

6. Zhao Y, Jiang C, He J, Guo Q, Lu J, et al. (2017) Multifunctional Dextran SulfateCoated Reconstituted High Density Lipoproteins Target Macrophages and Promote Beneficial Antiatherosclerotic Mechanisms. Bioconjug Chem 28: 438-448. [Crossref]

7. Kumara JBV, Ramakrishna S, Madhusudhan B (2017) Preparation and characterisation of atorvastatin and curcumin-loaded chitosan nanoformulations for oral delivery in atherosclerosis. IET Nanobiotechnol 11: 96-103. [Crossref]

8. Kumara JBV, Basavaraj M (2015) Formulation and evaluation of atorvastatin calcium loaded chitosan nanoparticles. Int J Pharm Bio Sci 6: 50-58.

9. Basavaraj M (2009) Potential benefits of flaxseed in health and disease-A perspective. Agriculturae Conspectus Scientificus 74: 67-72.

10. Prasad K, Jadhav A (2016) Prevention and treatment of atherosclerosis with flaxseedderived compound secoisolariciresinol diglucoside. Curr Pharm Des 22: 214-220. [Crossref]

11. Leyla PK, Ilhami G (2017) Inhibition Effects of Some Lignans on Carbonic Anhydrase, Acetylcholinesterase and Butyrylcholinesterase Enzymes. Rec Nat Prod 11: 65586561 .

12. Francis AA, Deniset JF, Austria JA, LaValleé RK, Maddaford GG, et al. (2013) Effects of dietary flaxseed on atherosclerotic plaque regression. Am J Physiol Heart Circ Physiol 304: H1743-H1751. [Crossref]

Copyright: (C2017 Bhat VS. This is an open-access article distributed under the terms of the Creative Commons Attribution License, which permits unrestricted use, distribution, and reproduction in any medium, provided the original author and source are credited. 